\title{
Genome report of three novel bacteriophages targeting different multidrug resistant clinical isolates (Escherichia, Klebsiella and Salmonella) from Enterobacteriaceae family
}

Roshan Nepal ( $\nabla$ roshan.nepal@adelaide.edu.au )

The University of Adelaide https://orcid.org/0000-0002-5469-3397

Ghais Houtak

The University of Adelaide

Sumeena Karki

Tribhuvan University

Gunaraj Dhungana

Tribhuvan University

Sarah Vreugde

The University of Adelaide

Rajani Malla

Tribhuvan University

\section{Research Article}

Keywords: Bacteriophage, Escherichia phage, Klebsiella phage, Salmonella phage, Virulent phage, Phage therapy, Enterobacteriaceae

Posted Date: March 21st, 2022

DOI: https://doi.org/10.21203/rs.3.rs-1363627/v2

License: (c) (i) This work is licensed under a Creative Commons Attribution 4.0 International License. Read Full License 
1 Genome report of three novel bacteriophages targeting different multidrug resistant

2 clinical isolates (Escherichia, Klebsiella and Salmonella) from Enterobacteriaceae family 3

4 Roshan Nepal ${ }^{1,2^{*}}$, Ghais Houtak ${ }^{1,2}$, Sumeena Karki ${ }^{3}$, Gunaraj Dhungana ${ }^{3}$, Sarah Vreugde ${ }^{1,2}$, $5 \quad$ Rajani Malla ${ }^{3}$

6

$7 \quad{ }^{1}$ Faculty of Health and Medical Sciences, The University of Adelaide, Adelaide, Australia.

$8{ }^{2}$ Department of Surgery-Otolaryngology Head and Neck Surgery, The Basil Hetzel Institute

9 for Translational Health Research, Central Adelaide Local Health Network, South Australia, 10 Australia.

$11{ }^{3}$ Central Department of Biotechnology, Tribhuvan University, Kirtipur, Nepal.

12

13

14

15

$16 *$ Correspondence:

17 roshan.nepal@adelaide.edu.au

18 Faculty of Health and Medical Sciences, The University of Adelaide

19 Adelaide, Australia.

20

21 Keywords:

22 Bacteriophage; Escherichia phage; Klebsiella phage, Salmonella phage, Virulent phage;

23 Phage therapy; Enterobacteriaceae

24

\section{Running title:}

26 Genome Report of Three Novel Phages 
Application of bacteriophages (phages) to treat complex multidrug resistant bacterial infection is gaining traction because of its efficacy and universal availability. However, as phages are specific to their host, a diverse collection of locally isolated phage from various geographical locations is required to formulate a wide host range phage cocktail. Here, we report morphological and genomic features of three newly isolated phages from river water of the urban region in Kathmandu, Nepal, targeting three different bacteria (Escherichia coli, Klebsiella pneumoniae and Salmonella spp.) from the Enterobacteriaceae family. Morphological identification and genome analysis indicated that two phages (Escherichia phage vB_EcoM_TU01 and Klebsiella phage vB_KpnP_TU02) were strictly lytic and free from integrases, virulence factors, toxins and known antimicrobial resistance genes whereas Salmonella phage vB_SalS_TU03 was possibly a temperate phage. The genomic features of these phages indicate that natural phages are capable of lysing pathogenic bacteria and have potential to be used therapeutically.

\section{Data availability}

The annotated genome assembly of Escherichia phage vB_EcoM_TU01, Klebsiella phage vB_KpnP_TU02, Salmonella virus vB_SalS_TU03 is available through GenBank accession MZ560701, MZ560702 and MZ560703 respectively. In addition, fastq file pertaining to raw sequence data is deposited at NCBI and is available through BioProject accession PRJNA383466 and Sequence Read Archive (SRA) identifiers SRR5460626, SRR5460625, SRR5460624 respectively.

Highlights

- Three novel phages exhibiting lytic activity against drug-resistant Escherichia coli, Klebsiella pneumoniae and Salmonella spp. were isolated and sequenced.

- Two of them (Escherichia phage vB_EcoM_TU01, Klebsiella phage vB_KpnP_TU02) were strictly virulent and belonged to Myoviridae (morphotype A2) while Salmonella virus vB_SalS_TU03 belonged to Siphoviridae (morphotype B1) family.

- All three phages did not encode any known lysogeny module, virulent factors and 


\section{Introduction}

62

63

64

65

66

67

68

69

Enterobacteriaceae are a large family of Gram-negative rod-shaped facultatively anaerobic bacteria comprising a wide range of pathogens such as Klebsiella, Salmonella, Escherichia, Enterobacter, Citrobacter, Shigella and more. These pathogens are associated with considerable morbidity and mortality on compromised hosts and can cause life-threatening illnesses like, septicaemia, haemolytic uremic syndrome, gastroenteritis, meningitis, and pneumonia in healthy individuals [1]. These infections are usually treated with antibiotics, but lately, most human associated pathogens are becoming increasingly resistant to antibiotics, thereby limiting the effectiveness of the antibiotic treatment. Furthermore, the emergence of carbapenem-resistant Enterobacteriaceae is a concern as there is no therapy or vaccines available to prevent acquisition of infection with multidrug resistant (MDR) strains. As current antibiotic therapies are ineffective to treat such infections or eliminate once infected, alternative approaches are sought in the management of MDR infections.

Bacteriophage (also known as phage) is a virus that infects bacterial cells but leaves eukaryotes unscathed. Because of its host specificity, phages can be used to kill bacteria without harming other cells. In the last decade, the application of phage therapeutically has been gaining widespread attention because of its specificity and efficacy against MDR bacterial pathogens [2]. Phage therapy uses 'strictly' lytic phages or its derivatives to kill pathogenic bacteria. Although phage therapy is not novel and had been employed shortly after the discovery of phages around 1920s [3], invention of antibiotics curbed the widespread usage of phages therapeutically as antibiotics were more effective against a broad spectrum of bacteria and were deemed more lucrative to the pharmaceutical industry. As mentioned before, the recent emergence of multidrug resistant 'superbugs' has rekindled the interest in phage therapy. Studies have shown that phage therapy can be used as an alternative biocontrol agent or adjuvant therapy to antibiotics in human and animals [4-8].

However, the efficacy of phage therapy targeting the pathogen of interest still has room for improvement. As phages are highly specific in regard to infecting their host, extending up to the level of bacterial strains, phages isolated from geographically same region as the bacterial host would have a higher probability of infecting the bacterial strain of interest due to the evolutionary adaptations [9]. Therefore, a local 'phage bank' comprising various phages isolated in the same region as bacterial pathogens of interest would facilitate a more effective strategy for the use of phage therapy. Since most of the genes in phage genome is yet 
'hypothetical', a comprehensive database reporting phage genome from different geolocations and clinical isolates is essential to study the co-evolution between phage and bacteria. As such, genome report provides invaluable information that can be useful in elucidating 'conserved and unknown' functions in phage genomes. Furthermore, the use of genomics and phenotyping of phages and their host could improve the efficacy of phage therapy in the future in regard to the choice of phage for the pathogen of interest. In line with the aim of expanding phage research, previously, we reported phages exhibiting lytic activity against multidrug resistant Pseudomonas and Klebsiella [10] and also studied pharmacokinetics and pharmacodynamics of our Klebsiella phage Kp_Pokalde_002 in a mouse model [11]. Here, we report the isolation, genome analysis and taxonomic position of three newly isolated phages targeting MDR human pathogens: Escherichia coli, Klebsiella pneumoniae and Salmonella spp. from Enterobacteriaceae family.

\section{Materials and methods}

\subsection{Bacterial strain}

Three multidrug resistant clinical isolates of $E$. coli $(\mathrm{N}=1), K$. pneumoniae $(\mathrm{N}=1)$ and Salmonella spp. $(\mathrm{N}=1)$ were collected from the Microbiology Laboratory, Tribhuvan University Teaching Hospital, Kathmandu, Nepal. The clinical isolates were confirmed to be MDR by AMR testing in the microbiology department of the hospital and used as hosts for isolation and amplification of phages. Nutrient agar (NA, agar $=1.5 \%$, HiMedia, India) was used to grow fresh overnight culture (at $37^{\circ} \mathrm{C}$ ) from glycerol stock and Luria-Bertani broth (HiMedia, India) was used to propagate the host bacterium for phage isolation and amplification.

\subsection{Phage manipulation: Isolation, purification and amplification}

A water sample was collected from the Bagmati river, Kathmandu, Nepal flowing through the urban region of the city which is heavily polluted by untreated sewers and industrial waste [12]. Phages were isolated using Double Layer Agar Assay (DLAA) as described previously with some modifications [10]. Briefly, the water sample was centrifuged at $3220 \times \mathrm{g}$ (Centrifuge 5810R, Eppendorf, Hamburg, Germany) for 10 minutes to pellet down the debris and subsequently the supernatant was filtered through a $0.45 \mu \mathrm{m}$ and $0.22 \mu \mathrm{m}$ pore-size Whatman $^{\mathrm{TM}}$ syringe filter (Sigma-Aldrich, Missouri, United States). One milliliter filtrate was 
mixed with $100 \mu \mathrm{l}$ exponentially growing host bacteria $\left(\mathrm{OD}_{600}=0.5\right)$ and left at room temperature $(10 \mathrm{~min})$ for phage adsorption. Three milliliter semisolid top agar (Tryptic Soya Agar (TSA), agar $=0.4 \%$, stored at $\left.=50^{\circ} \mathrm{C}\right)($ HiMedia, India) was added to the mixture, mixed well by swirling and poured on to the surface of previously prepared bottom agar (TSA, agar $=1.0 \%$, HiMedia, India). After overnight incubation at $37^{\circ} \mathrm{C}$, the plates were examined for the presence of phages in the form of plaques. A single isolated clear plaque was cut and dissolved in $1.0 \mathrm{~mL}$ of Sodium chloride-Magnesium sulfate $(\mathrm{SM})$ buffer $(10 \mathrm{mM}$ Tris- $\mathrm{HCl}, 10 \mathrm{mM}$ $\mathrm{MgSO}_{4} .7 \mathrm{H}_{2} \mathrm{O}, 2 \%$ gelatin and $100 \mathrm{mM} \mathrm{NaCl}, \mathrm{pH}$ 7.5). Subsequently, the phage was purified by performing three rounds of DLAA from a single isolated plaque.

\subsection{Phage characterization}

\subsubsection{Transmission electron microscopy}

140 High titre purified phage lysates were fixed with fixative $(2.5 \%$ glutaraldehyde and $2 \%$ paraformaldehyde prepared in 0.1 M sodium phosphate buffer ( $\mathrm{pH}$ 7.2)). For fixation, equal volume of phage lysate and fixative were added, mixed and left overnight. The next day, the fixed phages were subjected to high-speed centrifugation $(35,000 \times \mathrm{g})$ for 3 hours. Per sample $10.0 \mu \mathrm{L}$ fixed phage lysate was deposited on a separate 300 mesh carbon-coated copper grid. The copper grid was then flooded with $2 \%(\mathrm{w} / \mathrm{v})$ uranyl acetate $(\mathrm{pH} 4.5)$ for 2 minutes. The copper grid was dried and examined in JEM-2100F Transmission Electron Microscope (JEOL, USA, $200 \mathrm{KV}$ ) under different magnifications. TEM micrographs were processed using ImageJ 1.50i (https://imagej.nih.gov/ij) [13].

\subsubsection{Genomic DNA extraction, sequencing and annotation}

Phage genomic DNA (gDNA) was isolated using Phage DNA Isolation Kit (Norgen Biotek Corp., Ontario, Canada. Cat. \#46800) per manufacturer's instructions. Qualitative and quantitative control were performed using conventional electrophoresis and Qubit ${ }^{\circledR} 2.0$

154 Fluorometer (ThermoFisher Scientific, USA) respectively. Five microliter gDNA of each sample was loaded on 1\% agarose gel and run for 30 minutes at 110 Volt. Also, $1.0 \mu 1$ of each sample was loaded in NanoDrop 8000 (ThermoFisher Scientific, USA) for determining A260/280 ratio and Qubit ${ }^{\circledR} 2.0$ for determining concentration of gDNA. 
161 Covaris shearing that generated dsDNA fragments with $3^{\prime}$ or $5^{\prime}$ overhangs. The fragments were then subjected to end-repair. The ligated products were purified using SP beads supplied in the kit. The size-selected product was PCR amplified as described in the protocol. The amplified library was analyzed in Bio-analyzer 2100 (Agilent Technologies, USA) using High Sensitivity (HS) DNA chip as per manufacturer's instructions. After obtaining the Qubit ${ }^{\circledR}$ concentration for the library and the mean peak size from Bio-analyser profile (Figure S1A-C), the library was loaded onto Illumina HiSeq 2000/2500 (Illumina, USA) for cluster generation and sequencing. The cluster generated were assembled using CLC Genomics Workbench 6.0 (Qiagen, USA) at default parameters (Minimum contig length: 200, Automatic word size: Yes, Perform scaffolding: Yes, Mismatch cost: 2, Insertion cost: 3, Deletion cost: 3, Length fraction: 0.5, Similarity fraction: 0.8). Phage genomes were annotated for coding DNA sequences (CDS), tRNA, virulence factors, toxins, antimicrobial resistance genes (ARGs) and drug targets using the Pathosystems Resource Integration Center (PATRIC 3.6.12) webtool (https://www.patricbrc.org/) $[14,15]$ using viruses (taxid = 10239) as the reference database. A circular map of the phage genome was generated using CGview server (http://cgview.ca/) [16], and a phylogenetic tree was constructed BLASTing the query sequence against NCBI database using neighbor-joining method. Only the 10 most common phages were included in the phylogenetic analysis. The tree was further visualized using ggtree package in $\mathrm{R}$ 4.1.1 (https://www.R-project.org/). The lifestyle, order, family and host of the phages were computationally predicted through PhageAI (https://phage.ai/) [17].

\section{Results and discussion}

184 Three phages, viz: Escherichia phage vB_EcoM_TU01 (hereafter vB_EcoM_TU01), 185 Klebsiella phage vB_KpnM_TU02 (hereafter vB_KpnM_TU02) and Salmonella phage vB_SalS_TU03 (hereafter vB_SalS_TU03) targeting multidrug resistant clinical isolates of $E$. coli, K. pneumoniae and Salmonella spp. were isolated from the water sample collected from the Bagmati river (Figure 1A, 1C, 1E). TEM revealed that among three phages, two (vB_EcoM_TU01, vB_KpnM_TU02) were from the Myoviridae family whereas vB_SalS_TU03 belonged to Siphoviridae family (Figure 1B, 1D, 1F and Table 1). All phages were tailed phages (Order $=$ Caudovirales) and consist of a linear double-stranded DNA (dsDNA) genome with gene density of approximately 1.7 genes/kilo-basepairs which is much higher that of the bacterial host (0.5-1.0 genes/kilo-basepairs) [18]. The CDS coverage of all 
Table 1 | Classification of phages according to ICTV* guidelines (ICTV $9^{\text {th }}$ report) based on transmission electron micrograph.

\begin{tabular}{lllll}
\hline Phage & Capsid $\left(\right.$ in $\left.\mathbf{n m}^{\wedge}\right)$ & Tail $\left(\mathbf{W} \times \mathbf{L}\right.$, in $\left.\mathbf{n m}^{\wedge}\right)$ & Shape & Family (Morphotype $\left.{ }^{\sharp}\right)$ \\
\hline vB_EcoM_TU01, & $82 \times 108$ & $19 \times 111$ & Elongated & Myoviridae (A2) \\
\hline vB_KpnM_TU02 & $82 \times 99$ & $25 \times 109$ & Elongated & Myoviridae (A2) \\
\hline vB_SalS_TU03 & 63 & $9 \times 106$ & Icosahedral & Siphoviridae (B1) \\
\hline
\end{tabular}

* ICTV $=$ The International Committee on Taxonomy of Viruses. ${ }^{\wedge} \mathrm{nm}=$ nanometre.

\# Morphotypes are based on classification by Ackermann [19]

197

198

199

200

201

202

203

204

205

206

207

208

209

210

211

212

213

214

215

216

217

218

219

220

The genome of vB_EcoM_TU01 was 169,046 bp with a G+C content of 37.42\% [lower than that of its host $E$. coli $(\sim 50.6 \%)$ ] encoding 286 proteins (Figure 2$)$. The average length of genes was 566 bp with a CDS coverage of 95.9\%. Furthermore, vB_EcoM_TU01 encoded 2 transferRNAs (tRNA) (tRNA-Met-CAT and tRNA-Arg-TCT). Regarding the gene function, 83.2\% (238/286), were functional of which 5.6\% (16/286) had a Gene Ontology (GO) assigned function, and the remaining 16.8\% (48/286) were hypothetical. Similarly, the genome of vB_KpnM_TU02 was 166,230 bp with a G+C content of 38.34\% [lower than that of its host K. pneumoniae $(\sim 57 \%)$ ] and encoded 294 proteins (Figure 3 ). The average gene size in vB_KpnM_TU02 was 540 bp with a CDS coverage of 95.6\%. The phage vB_KpnM_TU02 also encoded 15 tRNAs (tRNA-Thr-TGT, tRNA-Leu-TAA, tRNA-Arg-TCT, tRNA-MetCAT, tRNA-Pro-TGG, tRNA-Gly-TCC, tRNA-Trp-CCA, tRNA-Ile-GAT, tRNA-Ser-TGA, tRNA-His-GTG, tRNA-Gln-TTG, tRNA-Met-CAT, tRNA-Asn-GTT, tRNA-Lys-TTT and tRNA-Tyr-GTA). Out of 294 encoded proteins, 110 (37.4\%) were functional, and 184 (62.6 $\%)$ were hypothetical, whereas only 11 (3.7\%) encoded proteins had GO assigned function. Further, the genome of vB_SalS_TU03 was 41,756 bp with a G+C content of $47.06 \%$ [slightly lower than that of its host Salmonella $(\sim 52.2 \%)]$ and encoded 71 proteins (Figure 4$)$. The average gene size in vB_SalS_TU03 was 562 bp with a CDS coverage of 95.7\%. Out of 71 encoded proteins, $45(63.4 \%)$ aligned with the functional protein whereas $26(36.6 \%)$ were hypothetical. Only 2 out of 71 (2.8\%) encoded proteins had GO assigned function.

Although the functions of tRNA in phages remain elusive, it is believed that more tRNA corresponds to increased virulence of the phage as it facilitates a more robust integration of the phages [20,21]. Since two of our phages encoded multiple tRNAs, it is more likely that these 
phages are virulent (lytic) and thus more suitable for therapeutic purposes. The 'functional' proteins include proteins involved in DNA packaging, transcription, replication, regulation, lysis and structural proteins whereas 'hypothetical' proteins are coding DNA sequences (CDS) with unknown functions. All the three phage genomes were free from genes encoding known toxins, antibiotic resistant genes (ARGs), virulent factors (VFs) of bacterial origin and lysogenic markers such as integrase, recombinase, repressor/anti-repressor protein, and excisionase. However, the in-silico tool we used (phageAI) only categorized vB_EcoM_TU01 and vB_KpnM_02 as virulent/lytic with high confidence (96.34\% and 99.27\% respectively) whereas vB_SalS_TU03 was tagged as temperate/lysogenic with a low confidence of $57 \%$. The substantial number of hypothetical proteins in all phages clearly indicates that phages carry numerous genes that are yet to be characterized, and whose function is yet to be discovered. The detailed information about the genomes of all three phages and their respective lifestyle is summarized in Table 2. These results suggest that vB_EcoM_TU01 and vB_KpnM_02 could potentially be used as therapeutic phages against multidrug resistant E. coli and K. pneumoniae whereas vB_SalS_TU03 would less likely succeed in lysing its host.

Further, comparing the phage genome in the NCBI database using nucleotide BLAST (nBLAST) revealed that the phage vB_EcoM_TU01 was closely related to a T4-like lytic Escherichia phage vB_EcoM_JS09 (NCBI accession $=$ KF582788, query coverage $=99 \%$, percent identity $=98.04 \%$ ) isolated in China from the sewage of a swine factory. Similarly, phage vB_KpnM_TU01 was similar to a lytic Klebsiella phage JD18 (NCBI accession = KT239446, query coverage $=96 \%$, percent identity $=97.89 \%$ ) isolated in China. Further, phage vB_SalS_TU03 was closest to lytic Salmonella phage LSPA1 (NCBI accession = KM272358, query coverage $=93 \%$, percent identity $=99.17 \%$ ) isolated in China from a hospital sewage [22]. These analyses indicate that our phages were novel, but highly similar to the phages isolated in neighbouring China around the same time and might have a very similar host range. Phylogenetic relatedness of all three phages against ten most common phages and their percent identity is elaborated in Figure 5. It is noted that, among 10 most common hits, phylogenetics reveal that vB_EcoM_TU01 is also closely related to Shigella phages (also an Enterobacteriaceae). Although more study is required, we can arbitrarily predict that phages isolated against different genus of bacteria have higher degree of similarity between them. This may explain (although not studied in this research) why some phages are polyvalent (showing inter-genus or even inter-order infectivity) and show expansive host spectrum [23-26]. This property thus holds immense applicability if further study is performed to determine the 
255 mechanism of phage infection and identify the factors/proteins/enzymes that determine phage256 bacteria specificity. 
Table 2 | Genomic and protein features of three novel phages targeting multidrug resistant Escherichia coli, Klebsiella pneumoniae and Salmonella spp. clinical isolates.

\begin{tabular}{|c|c|c|c|}
\hline Features & $\begin{array}{l}\text { Escherichia phage } \\
\text { vB_EcoM_TU01 }\end{array}$ & $\begin{array}{l}\text { Klebsiella phage } \\
\text { vB_KpnM_TU02 }\end{array}$ & $\begin{array}{l}\text { Salmonella phage } \\
\text { vB_SalS_TU03 }\end{array}$ \\
\hline NCBI accession & MZ560701 & MZ560702 & MZ560703 \\
\hline \multicolumn{4}{|l|}{ Genomic features } \\
\hline Length (in base pairs) & $169,046 \mathrm{bp}$ & $166,230 \mathrm{bp}$ & $41,756 \mathrm{bp}$ \\
\hline Guanine-cytosine $(\mathrm{G}+\mathrm{C})$ content & $37.42 \%$ & $38.34 \%$ & $47.06 \%$ \\
\hline Total CDS/ORFs & 286 & 294 & 71 \\
\hline tRNAs & 2 & 15 & 0 \\
\hline Gene density (per kbp) & 1.69 & 1.77 & 1.70 \\
\hline Average gene size (in bp) & 566 & 540 & 562 \\
\hline CDS coverage & $95.9 \%$ & $95.6 \%$ & $95.7 \%$ \\
\hline \multicolumn{4}{|l|}{ Protein feature } \\
\hline Hypothetical proteins & $48(16.78 \%)$ & $184(62.59 \%)$ & $26(36.62 \%)$ \\
\hline Functional proteins & $238(83.22 \%)$ & $110(37.41 \%)$ & $45(63.38 \%)$ \\
\hline Proteins with GO assignments & $16(5.60 \%)$ & $11(3.74 \%)$ & $2(2.82 \%)$ \\
\hline \multicolumn{4}{|l|}{ Other features/genes } \\
\hline Transporter genes $(\mathrm{Ref}=\mathrm{TCDB})$ & 5 & 0 & 0 \\
\hline Drug target genes $($ Ref = DrugBank) & 3 & 0 & 0 \\
\hline Order & Caudovirales & Caudovirales & Caudovirales \\
\hline Family & Myoviridae & Myoviridae & Siphoviridae \\
\hline Genus (Ref = PhageAI, NCBI) & Mosigvirus & Jiaodavirus & Jerseyvirus \\
\hline Lifestyle $($ Ref $=$ PhageAI $)$ & Virulent $(\mathrm{C}=96 \%)$ & Virulent $(\mathrm{C}=99 \%)$ & Temperate $(\mathrm{C}=57 \%)$ \\
\hline \multicolumn{4}{|c|}{ 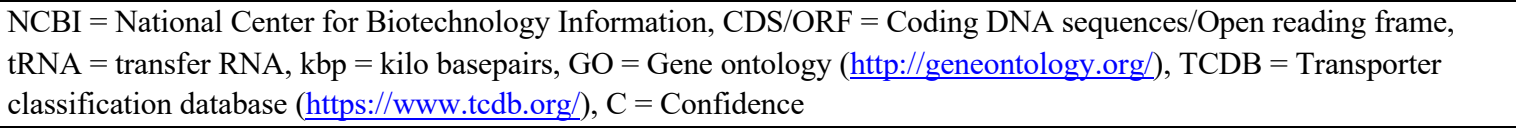 } \\
\hline
\end{tabular}

\section{Conclusion}

260 Three phages infecting multidrug resistant E. coli, K. pneumoniae and Salmonella spp. were isolated, sequenced and banked. Genome analysis indicated that two of them (Escherichia phage vB_EcoM_TU01 and Klebsiella phage vB_KpnP_TU02) were strictly lytic and free from integrases, virulence factors, toxins, and antimicrobial resistance genes. Although additional studies are required, the genomic features of these phages provide valuable insights into the possibility of using natural phages as biocontrol agents against multidrug resistant human pathogens. 


\section{CRediT author statement}

270 Conceptualization and funding acquisition: RM, RN. Methodology, investigation: RN, SK.

271 Analysis and visualization: RN, SK, GH. Supervision: RM. Writing - original draft: RN, SK,

272 GH. Writing - review \& editing: RN, GD, GH, SV.

273

274 Ethical approval

275 The study does not involve any human and/or animal subjects. The clinical isolates obtained

276 from hospital was deidentified, and no personally identifiable patient information was

277 disclosed to the researchers.

278

279 Acknowledgements

280 We are grateful to Asst/Prof. Sneh Lata Panwar and her lab members (SLS-JNU, New Delhi,

281 India), Dr. Gajender Saini (AIRF-JNU, New Delhi, India) for assisting with the TEM analysis

282 and Xcelris Labs Ltd., Ahmedabad, India for providing sequencing facility. We also extend

283 our gratitude to the staffs at Microbiology Laboratory, TUTH, Kathmandu, Nepal for kindly

284 providing MDR clinical isolates. This study was partially supported by Kathmandu Center for

285 Education and Research CAS \& TU Thesis Grant for M.Sc. students - 2015 granted to RN. We 286 declare no conflicts of interest.

287

288

289 
291 1. Donnenberg MS. Enterobacteriaceae. In: Bennett JE, Dolin R, Blaser MJ (editors).

292 Mandell, Douglas, and Bennett's Principles and Practice of Infectious Diseases (Eighth

293 Edition). Philadelphia: W.B. Saunders; 2015. pp. 2503-2517.e2505.

294 2. Pirnay J-P. Phage Therapy in the Year 2035. Frontiers in Microbiology 2020;11,

295 doi: 10.3389/fmicb.2020.01171.

296 3. d'Herelle F. Bacteriophage as a Treatment in Acute Medical and Surgical Infections. Bull N Y Acad Med 1931;7(5):329-348. PMID: 19311785, doi.

4. Petrovic Fabijan A, Lin RCY, Ho J, Maddocks S, Ben Zakour NL et al. Safety of bacteriophage therapy in severe Staphylococcus aureus infection. Nature Microbiology

300 2020;5(3):465-472, doi: 10.1038/s41564-019-0634-z.

$301 \quad 5 . \quad$ Schooley RT, Biswas B, Gill JJ, Hernandez-Morales A, Lancaster J et al. Development and Use of Personalized Bacteriophage-Based Therapeutic Cocktails To Treat a Patient with a Disseminated Resistant Acinetobacter baumannii Infection. Antimicrob Agents Chemother 2017;61(10):AAC.00954-00917, doi: 10.1128/aac.00954-17.

6. Ooi ML, Drilling AJ, Morales S, Fong S, Moraitis S et al. Safety and Tolerability of Bacteriophage Therapy for Chronic Rhinosinusitis Due to Staphylococcus aureus. JAMA Otolaryngology-Head \& Neck Surgery 2019;145(8):723-729, doi: 10.1001/jamaoto.2019.1191.

309 7. Waters EM, Neill DR, Kaman B, Sahota JS, Clokie MRJ et al. Phage therapy is 310 highly effective against chronic lung infections with Pseudomonas aeruginosa. Thorax 311 2017;72(7):666, doi: 10.1136/thoraxjnl-2016-209265.

$312 \quad 8$. Greene W, Chan B, Bromage E, Grose JH, Walsh C et al. The Use of

313 Bacteriophages and Immunological Monitoring for the Treatment of a Case of Chronic 314 Septicemic Cutaneous Ulcerative Disease in a Loggerhead Sea Turtle Caretta caretta. Journal of Aquatic Animal Health 2021;33(3):139-154, doi: 10.1002/aah.10130.

9. Hampton HG, Watson BNJ, Fineran PC. The arms race between bacteria and their phage foes. Nature 2020;577(7790):327-336, doi: 10.1038/s41586-019-1894-8.

10. Dhungana G, Regmi M, Paudel P, Parajuli A, Upadhyay $\mathbf{E}$ et al. Therapeutic

319 Efficacy of Bacteriophage Therapy to Treat Carbapenem-Resistant Klebsiella pneumoniae in 320 a Mouse Model. Journal of Nepal Health Research Council 2021;19(1), doi:

321 10.33314/jnhrc.v19i1.3282.

322 11. Dhungana G, Nepal R, Regmi M, Malla R. Pharmacokinetics and Pharmacodynamics of a Novel Virulent Klebsiella Phage Kp_Pokalde_002 in a Mouse Model. Frontiers in Cellular and Infection Microbiology, Original Research 2021;11(731), doi: 10.3389/fcimb.2021.684704.

12. Mishra BK, Regmi RK, Masago Y, Fukushi K, Kumar P et al. Assessment of Bagmati river pollution in Kathmandu Valley: Scenario-based modeling and analysis for sustainable urban development. Sustainability of Water Quality and Ecology 2017;9-10:6777, doi: 10.1016/j.swaqe.2017.06.001.

13. Schneider CA, Rasband WS, Eliceiri KW. NIH Image to ImageJ: 25 years of image analysis. Nature Methods 2012;9(7):671-675, doi: 10.1038/nmeth.2089.

14. Wattam AR, Abraham D, Dalay O, Disz TL, Driscoll T et al. PATRIC, the bacterial bioinformatics database and analysis resource. Nucleic Acids Research 2013;42(D1):D581-D591, doi: 10.1093/nar/gkt1099.

15. Brettin T, Davis JJ, Disz T, Edwards RA, Gerdes S et al. RASTtk: A modular and extensible implementation of the RAST algorithm for building custom annotation pipelines and annotating batches of genomes. Scientific Reports 2015;5(1):8365, doi: $10.1038 /$ srep08365. 
339 16. Stothard P, Wishart DS. Circular genome visualization and exploration using

340 CGView. Bioinformatics 2004;21(4):537-539, doi: 10.1093/bioinformatics/bti054.

341 17. Tynecki P, Guziński A, Kazimierczak J, Jadczuk M, Dastych J et al. PhageAI -

342 Bacteriophage Life Cycle Recognition with Machine Learning and Natural Language

343 Processing. bioRxiv 2020:2020.2007.2011.198606, doi: 10.1101/2020.07.11.198606.

344 18. Norwood DA, Sands JA. Physical map of the Clostridium difficile chromosome.

345 Gene 1997;201(1):159-168, doi: 10.1016/S0378-1119(97)00443-5.

346 19. Ackermann HW. Frequency of morphological phage descriptions in the year 2000.

347 Archives of Virology 2001;146:843-857, doi: 10.1007/s007050170120.

348 20. Bailly-Bechet M, Vergassola M, Rocha E. Causes for the intriguing presence of

349 tRNAs in phages. Genome Res 2007;17(10):1486-1495, doi: 10.1101/gr.6649807.

350 21. Maganha de Almeida Kumlien AC, Pérez-Vega C, González-Villalobos E,

351 Borrego CM, Balcázar JL. Genome analysis of a new Escherichia phage vB_EcoM_C2-3

352 with lytic activity against multidrug-resistant Escherichia coli. Virus Research

353 2022;307:198623, doi: https://doi.org/10.1016/j.virusres.2021.198623.

354 22. Zeng W, Mao P, Hong Y, Feng M, Xu Z et al. Complete Genome Sequence of the

355 Salmonella enterica Serovar Paratyphi A Bacteriophage LSPA1 Isolated in China. Genome Announcements 2015;3(1):e01011-01014, doi: 10.1128/genomeA.01011-14.

23. Gambino M, Nørgaard Sørensen A, Ahern S, Smyrlis G, Gencay YE et al. Phage S144, a New Polyvalent Phage Infecting Salmonella spp. and Cronobacter sakazakii.

359 International Journal of Molecular Sciences 2020;21(15):5196, doi: 10.3390/ijms21155196.

360 24. Hamdi S, Rousseau GM, Labrie SJ, Tremblay DM, Kourda RS et al.

361 Characterization of two polyvalent phages infecting Enterobacteriaceae. Scientific Reports

362 2017;7(1):40349, doi: 10.1038/srep40349.

363 25. Sui B, Han L, Ren H, Liu W, Zhang C. A Novel Polyvalent Bacteriophage

364 vB_EcoM_swi3 Infects Pathogenic Escherichia coli and Salmonella enteritidis. Frontiers in Microbiology, Original Research 2021;12(1496), doi: 10.3389/fmicb.2021.649673. 26. Yu P, Mathieu J, Li M, Dai Z, Alvarez PJJ et al. Isolation of Polyvalent Bacteriophages by Sequential Multiple-Host Approaches. Applied and Environmental Microbiology 2016;82(3):808-815, doi: doi:10.1128/AEM.02382-15. 


\section{$371 \quad$ Figure legends}

\section{Figure 1 |}

373 Phage isolation using double layer agar assay and their transmission electron micrograph 374 (TEM). (A, C, E) Three plates showing different types of plaques. (B) TEM of Escherichia 375 phage vB_EcoM_TU01 (scale bar $=100 \mathrm{~nm}$ ), (D) TEM of Klebsiella phage vB_KpnM_TU02 376 (scale bar $=100 \mathrm{~nm}),(\mathrm{F})$ TEM of Salmonella phage vB_SalS_TU03 (scale bar $=20 \mathrm{~nm})$.
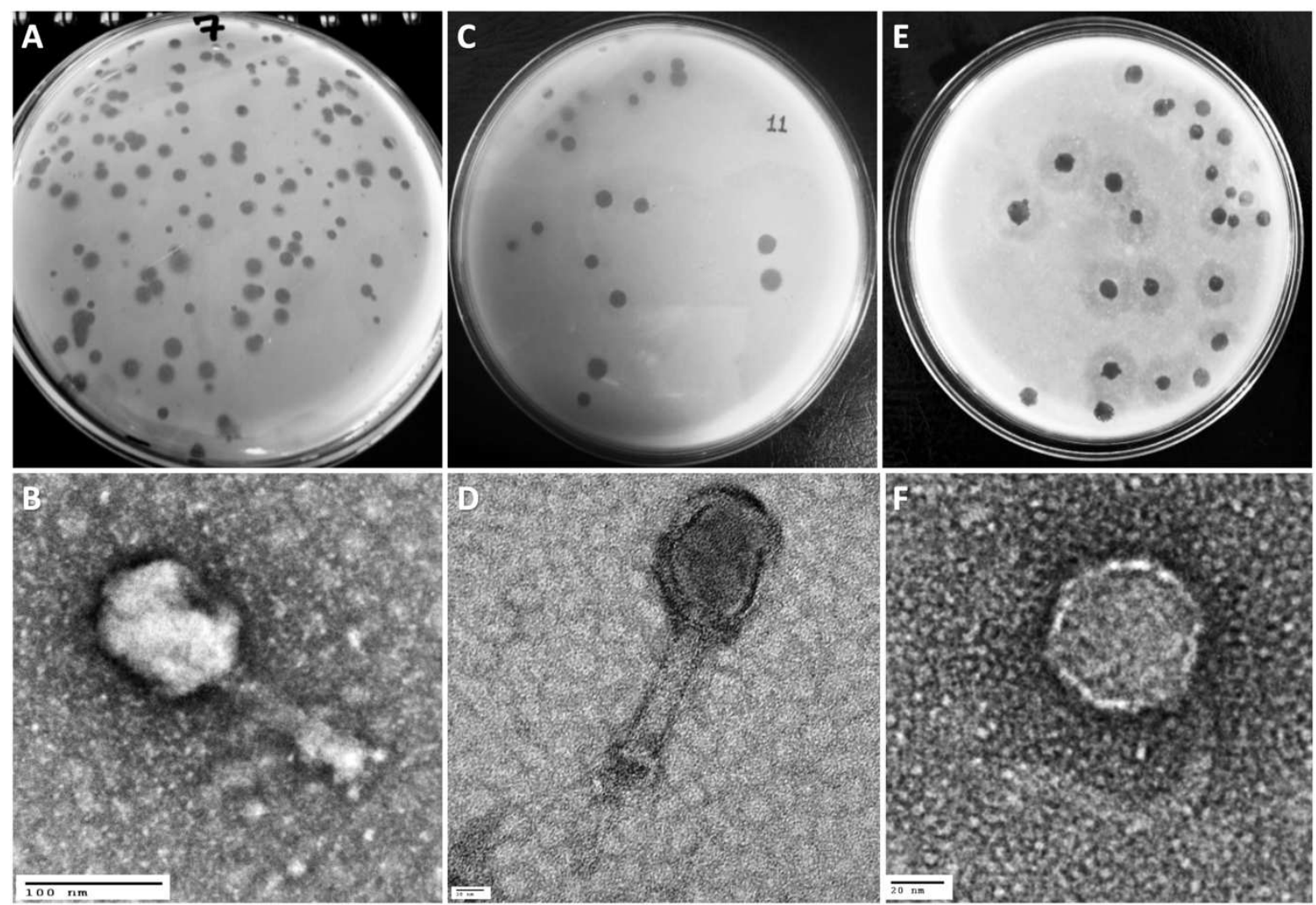


\section{Figure 2 |}

380 Genome organization of Escherichia phage vB_EcoM_TU01 targeting multidrug resistant

381 Escherichia coli clinical isolate. Predicted coding regions are shown by arrows indicating the 382 direction of the transcription.

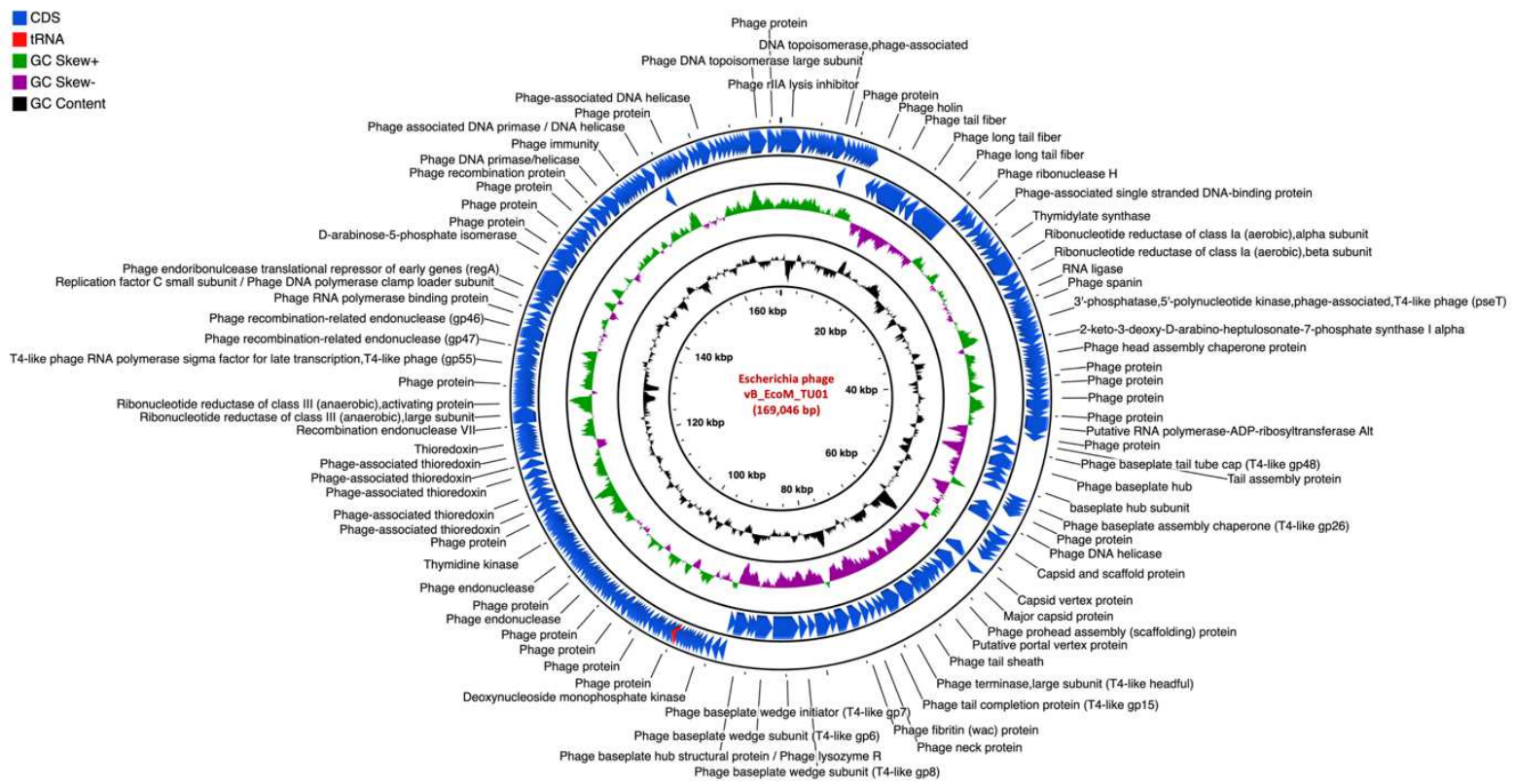




\section{Figure 3 |}

387 Genome organization of Klebsiella phage vB_KpnM_TU02 targeting multidrug resistant

388 Klebsiella pneumoniae clinical isolate. Predicted coding regions are shown by arrows 389 indicating the direction of the transcription.

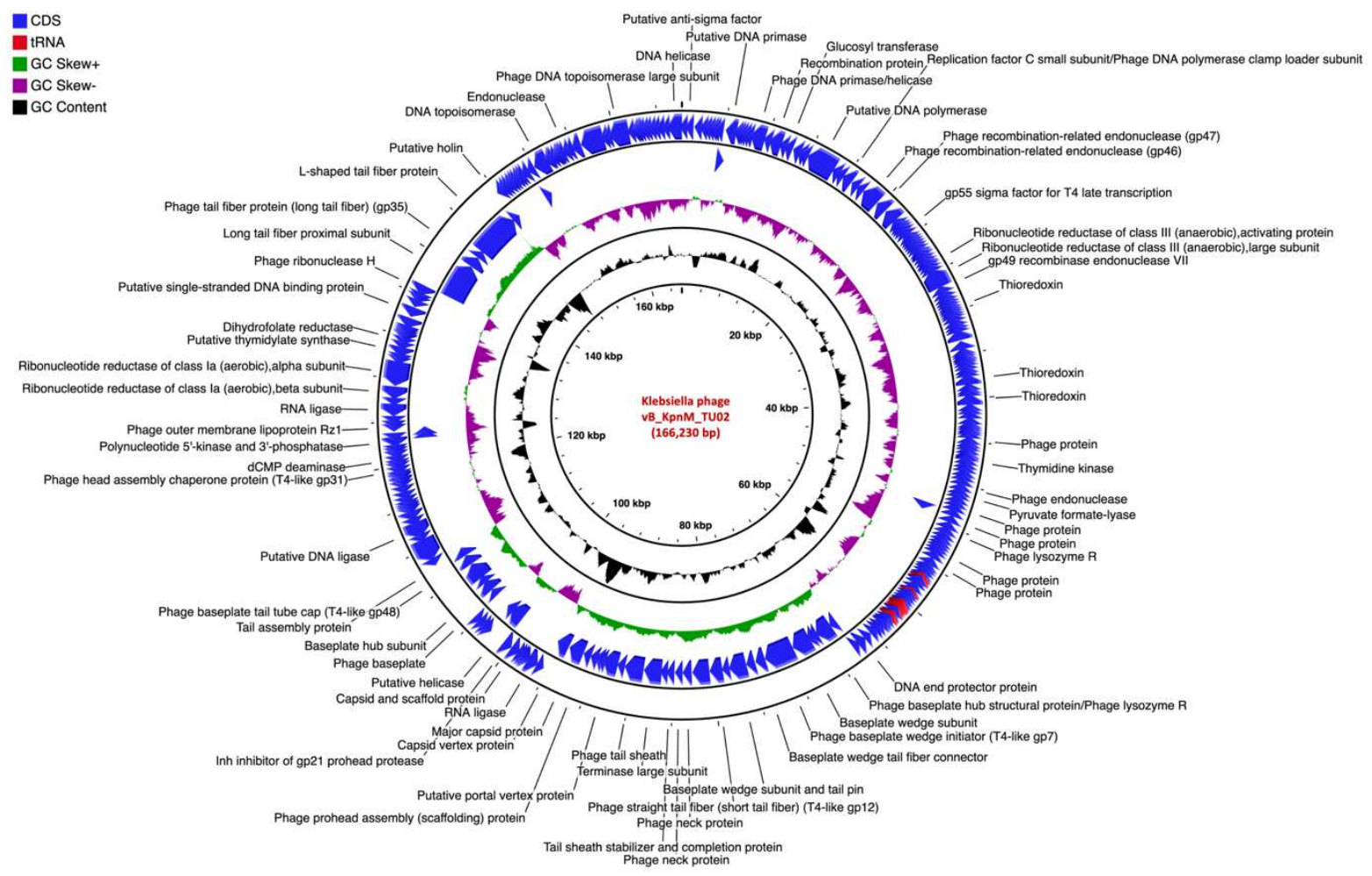




\section{$393 \quad$ Figure 4 |}

394 Genome organization of Salmonella phage vB_SalS_TU03 targeting multidrug resistant

395 Salmonella spp. clinical isolate. Predicted coding regions are shown by arrows indicating the 396 direction of the transcription.

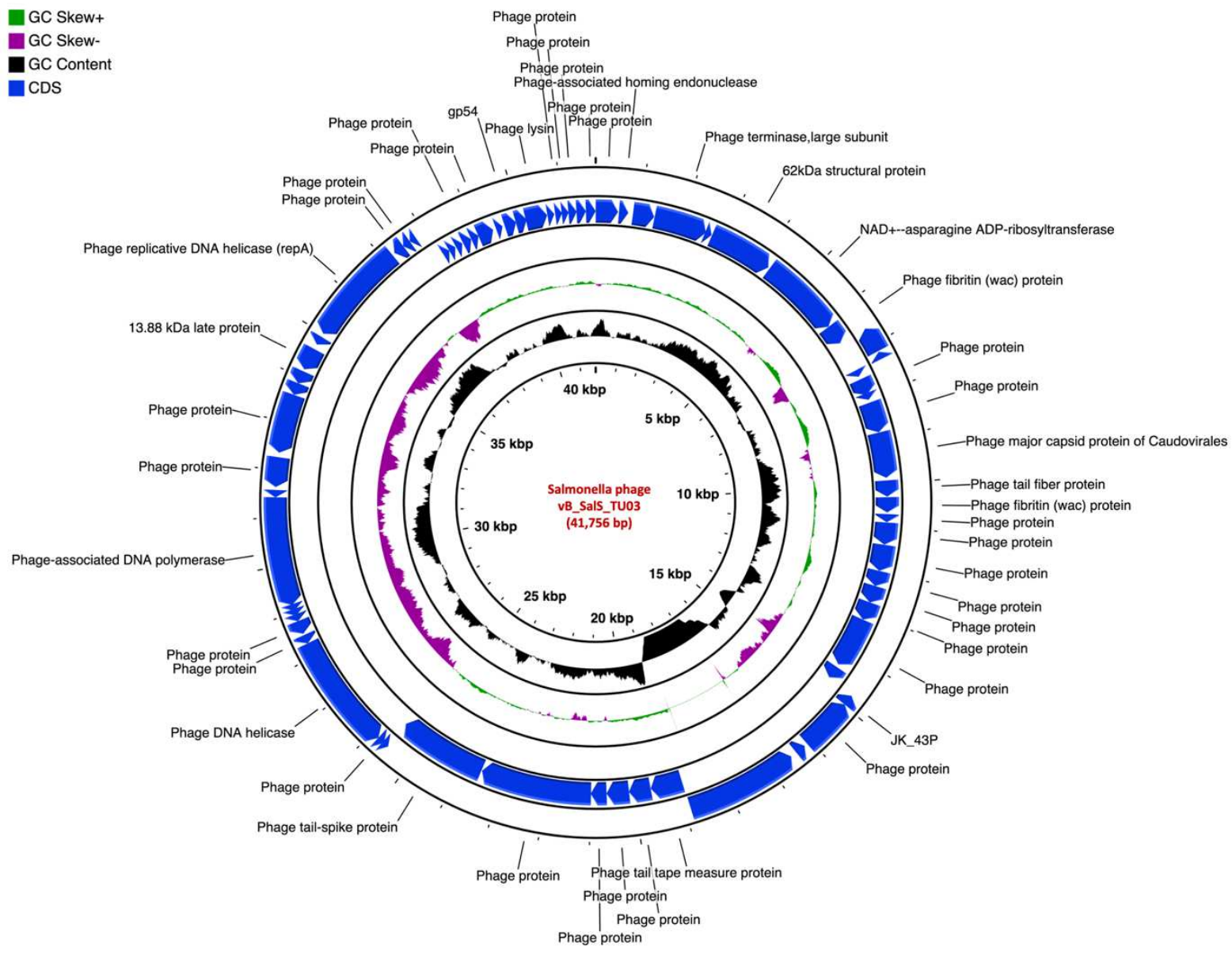


$400 \quad$ Figure 5 |

401 Phylogenetic relatedness of Escherichia phage vB_EcoM_TU01 (A), Klebsiella phage 402 vB_KpnM_TU02 (B) and Salmonella phage vB_SalS_TU03 (C) against most common phage 403 hits $(\mathrm{N}=10)$ in the NCBI database. The phylogenetic tree was constructed using neighbour404 joining method.
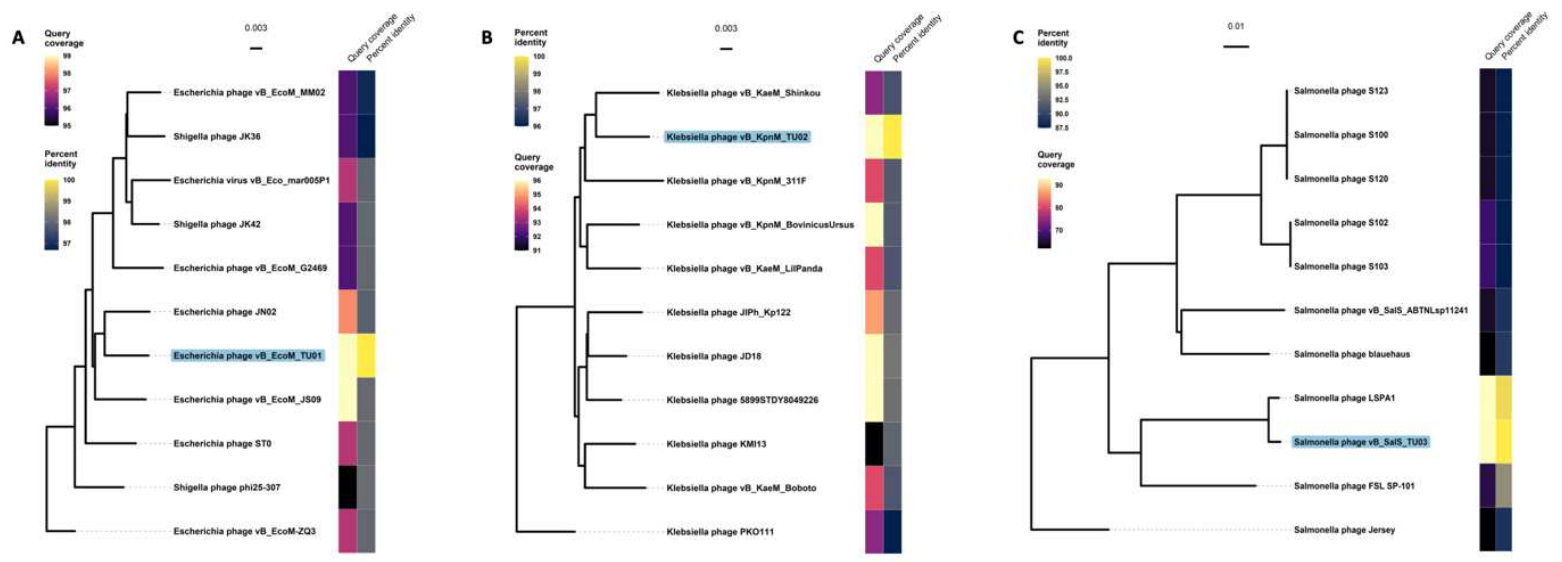

405

406

407 


\section{Supplementary Figure S1 |}

409 Bio-analyser profile of DNA library loaded in Agilient DNA HS chip. The mean sizes of the

410 fragmented libraries (gDNA) of Escherichia phage vB_EcoM_TU01 (A), Klebsiella phage 411 vB_KpnM_TU02 (B), and Salmonella phage vB_SalS_TU03 (C) were 630 bp, 616 bp and 675 412 bp respectively. (D) Genomic DNA of isolated phages. Marker = Hind III DNA ladder, Lane 4131 = Escherichia phage vB_EcoM_TU01, Lane 2 = Klebsiella phage vB_KpnM_TU02, Lane 3 $414=$ Salmonella phage vB_SalS_TU03. The numbers on the bottom indicate concentration of 415 genomic DNA in $\mathrm{ng} / \mu \mathrm{l}$ as indicated by Qubit 2.0 fluorometer. (E) Average gene length of 416 isolated phages.

417

A

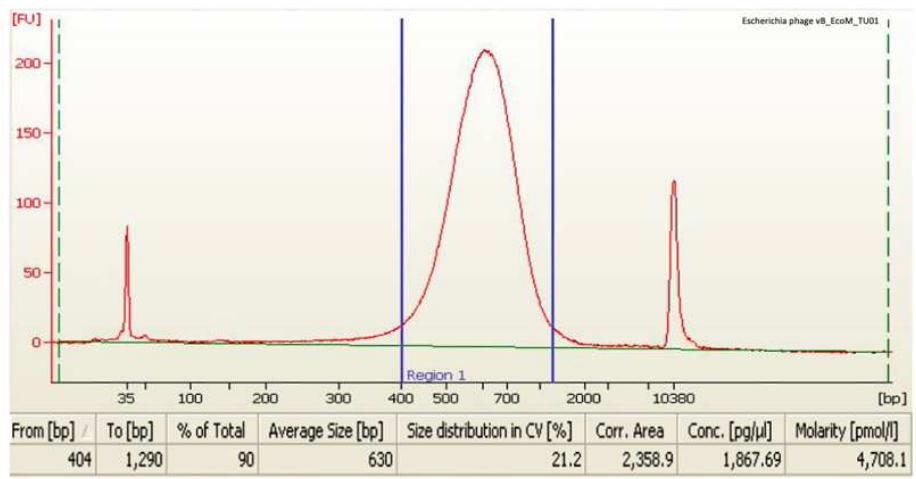

B

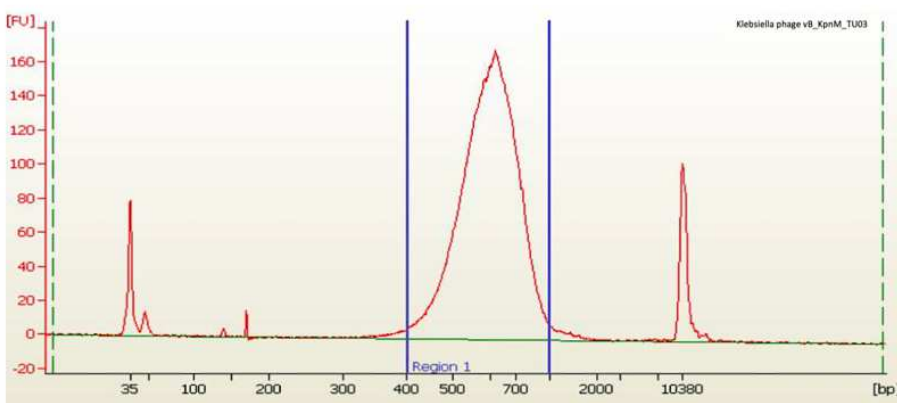

\begin{tabular}{|l|l|l|l|l|l|l|l}
\hline From [bp] & To [bp] & $\%$ of Total & Average Size [bp] & Size distribution in Cv [\%] & Corr. Area & Conc. [pg/ll] & Molarity [pmol/[] \\
\hline
\end{tabular}

C

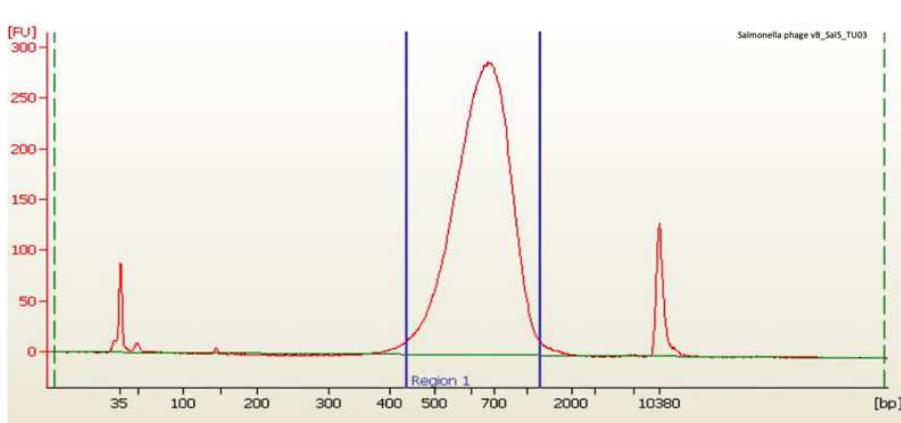

\begin{tabular}{|l|l|l|l|l|l|l|l|}
\hline From [bp] & To [bp] & $\%$ of Total & Average Size [bp] & Size distribution in CV [\%] & Corr. Area & Conc. [pg//l]] & Molarity [pmolil] \\
\hline
\end{tabular} $\begin{array}{lll}438 & 1,297 \quad 96\end{array}$
D

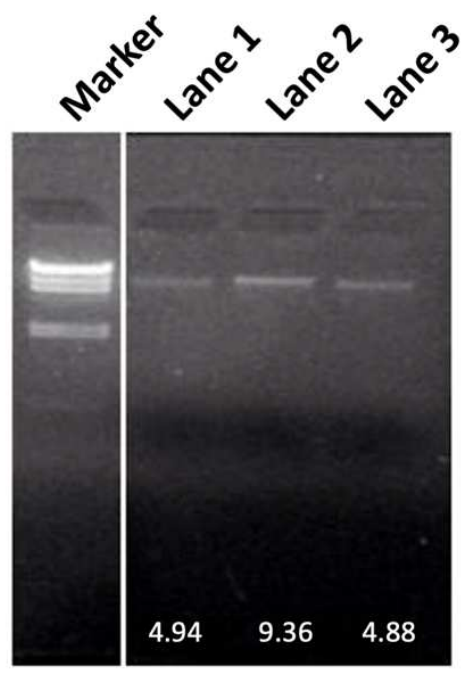

E

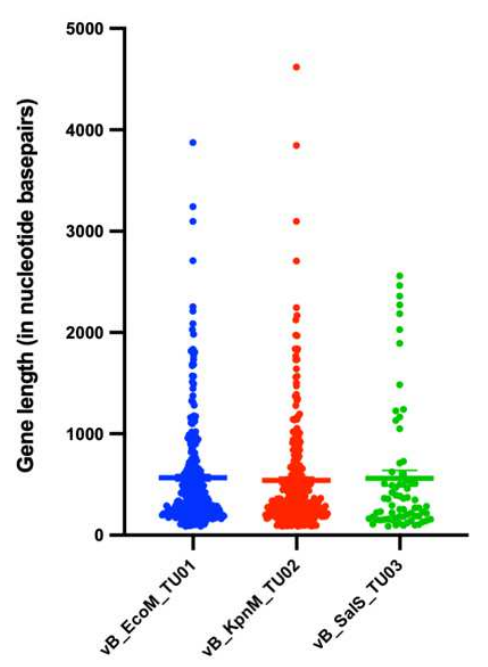


419 APPENDIX I

420 Abbreviations

421

422 MDR Multidrug Resistance

423 DLAA Double Layer Agar Assay

424 PCR Polymerase Chain Reaction

425 CDS Coding DNA Sequence

426 tRNA Transfer RNA

427 ARG Antibiotic Resistant Gene

428 PATRIC Pathosystems Resource Integration Center

429 NCBI National Center for Biotechnology Information

430 TEM Transmission Electron Microscopy

431 dsDNA Double-strained DNA

432 GO Gene Ontology

$433 \mathrm{G}+\mathrm{C} \quad$ Guanine and Cytosine

434 ORF Open Reading Frame 